\title{
Intersectionality: The Role of the Athletic Trainer in Providing Culturally Competent Patient-Centered Care
}

\author{
Rebecca M. Lopez, PhD, ATC, CSCS*; Samar Long, MSEd, AT, ATC†; Dani M. Moffit, PhD, LAT, ATC‡; and \\ Ashley K. Crossway, DAT, ATC** \\ *University of South Florida, Tampa Bay; TOhio State University, Columbus; ¥ldaho State University, Pocatell; **State University \\ of New York College, Cortland
}

Key Phrases

Cultural competence; intersectionality; healthcare disparities

\section{Correspondence}

Dr. Rebecca M. Lopez, University of South Florida,

13220 USF Laurel Dr. MDC 106, Tampa, FL 33612.

E-mail: rml@usf.edu

Twitter: @Rlopez1010

\section{Full Citation}

Lopez RM, Long S, Moffit DM, Crossway AK. Intersectionality: The role of the athletic trainer in providing culturally competent patient-centered care. Clin Pract Athl Train. $2021 ; 4(1): 8-16$.

https://doi.org/10.31622/2021/0004.1.2.

Submitted: September 25, 2020 Accepted: January 22, 2020.

Supplemental Infographic:

https://create.piktochart.com/output/4861 1749-my-visual

\section{INTRODUCTION}

O ne of the key components of evidence-based medicine is incorporating the patient's values and characteristics into the plan of care. As healthcare providers, athletic trainers should understand the importance of patient values and providing patient-centered care. However, this process of incorporating the patient's values into the care provided requires learning more about the patient as a whole, rather than solely obtaining information regarding the reason they sought care. This process of patient-centered care provides focus beyond the simple characteristics seen on the outside. Historically, patients who are of racial or ethnic minorities, such as those that are Black or indigenous persons of color (BIPOC) experience unconscious biases in medical care that lead to healthcare inequities. ' It has been documented in Black women that the intersection

Copyright $(\subset)$ by Indiana State University All rights reserved. ISSN Online 2577-8188 of gender and race can lead to further discrimination. ${ }^{1,2}$ Similarly, individuals of sexual and gender minorities have been marginalized and experience significant healthcare disparities. ${ }^{3}$ Individuals that identify as lesbian, gay, bisexual, transgender, queer, intersex, or other gender or sexual minority (LGBTQ+) experience healthcare disparities due to inadequate treatment, as well as avoidance of seeking care due to discrimination and stigmatization by healthcare providers. Similarly, patients who are of racial or ethnic minorities, such as primarily those that are BIPOC, have also experienced unconscious biases in medical care that lead to healthcare inequities. ${ }^{1}$ Studies on athletic trainers' perceptions of lesbian, gay, and bisexual (LGB) patients ${ }^{4}$ and LGBTQ+ patients 5 have shown that respondents overall had positive attitudes toward LGBTQ+ patients, however, this was largely dependent on clinicians being female and/or having a close friend or relative that identified as $L B G$ or $L G B T Q+.4,5$ To our knowledge, there are no published studies specific to the healthcare provided to BIPOC patients by athletic trainers. However, it is important not to compartmentalize our patients as belonging to one group or via a single-axis framework. This is highlighted by Crenshaw's work describing how the intersection of race and sex often results in the multidimensionality of the discrimination some individuals face. ${ }^{2}$ The intersection of race and sexual orientation and gender demonstrates that these factors are not mutually exclusive. ${ }^{2}$ Individuals that belong to more than one marginalized group (i.e. race, sexual orientation, and gender identity) may have negative experiences (i.e. discrimination, 
inadequate care) in healthcare due to belonging to a "dual minority".

Implicit biases, prejudices, and stigmatization faced by marginalized groups often negatively impact mental health; this is referred to as minority stress. ${ }^{6}$ The LGBTQ+ community, and other minority groups, find these experiences further lead to healthcare and health disparities. Understanding these various aspects of intersectionality as it relates to ethnic minorities, sexual minorities, and other marginalized groups is essential in providing culturally competent care. Therefore, the purpose of this commentary is to describe culturally competent care for LGBTQ+ patients impacted by intersectionality and healthcare disparities.

\section{INTERSECTIONALITY}

People who have multiple marginalized identities often experience additional barriers to equitable healthcare when compared to those with singular marginalized identities; this cumulative effect is attributed to the theory of intersectionality. ${ }^{2}$ Intersectionality is a theoretical framework that considers multiple social categories (i.e. race, ethnicity, gender, sexual orientation, socioeconomic status) intersecting at the micro level of individual experience to reflect multiple interlocking systems of privilege and oppression at the macro, social-structural level (i.e., racism, sexism, heterosexism).7 The phrase "women and minorities," often used together, implies these two identifiers are mutually exclusive, when in fact, these two categories often intersect. ${ }^{7}$ Additionally, minority has many different definitions. It can reference populations such as individuals in the LGBTQ+ community; people with mental and/or physical disabilities; or, when living in other countries, White people. ${ }^{7}$ For example, the multiple layers of intersecting categories can lead one to a low-income Black bisexual man with a physical disability.

Copyright $(\subset)$ by Indiana State University All rights reserved. ISSN Online 2577-8188
When considering the social determinants of health (i.e., socioeconomic status, gender, race, ethnicity, community), it is critical to consider how intersectionality can create complexity in treating patients. Inequities and/or unfair gaps are widened when a person falls into more than one category. Historically, White males with financial security receive the best medical care. ${ }^{7}$ Consider the Latino gay male who is not financially secure. The care he receives could change simply because of several distinct characteristics, in that he is gay, is a member of an ethnic minority, and is not financially secure. Social justice in healthcare is relevant, but those who tend to be most harmed by social inequalities are those who have multiple forms of discriminatory characteristics in their being. ${ }^{8}$ Part of the role of any healthcare provider is to know their patient; lack of understanding on potential barriers to care and discriminatory experiences by an athletic trainer can negatively affect the patient-provider relationship and lead to future health concerns of the patient. For example, athletic trainers should be concerned with their patients' health across the lifespan; if a patient has a negative experience with one healthcare provider, this can be a deterrent and prevent this patient from seeking care from another provider in the future. Therefore, understanding healthcare experiences may be moderated for patients who fall into more than one specific category and how this can impact their treatment and recovery. Further, this understanding becomes imperative for any athletic trainer to better understand the needs of the patient.

\section{MINORITY STRESS}

Health disparity research often focuses on an individual cultural group, often race or sexual orientation, as opposed to considering the whole person which exists at the center of multiple identities. ${ }^{1}$ For persons with multiple social disadvantages, both health and well-being are influenced in a variety of ways. ' Both 
intersectionality and minority stress theories are helpful frameworks for understanding how compounding experiences of racism and homophobia cause barriers to seeking care and mistrust in the patient-provider relationship. ${ }^{1}$ For example, an individual that is part of an ethnic minority and is also part of the LGBTQ+ community may experience adverse treatment that is commonly noted in each community, such as refusal of care or having expressed needs ignored or dismissed. Often, having a very negative experience while seeking care from a healthcare provider is unique in that the patient's hardships experienced in vastly differently communities converge.

The minority stress model evolved from Dr. llan Myers original work in 1995 to build the concept of a unique stress that sexual minority people (i.e., individuals in the LGBTQ+ community) experience as a result of stigma, prejudice, and discrimination due to their sexual identity. ${ }^{6}$ Lived experiences marked by hostile and stressful social environments contribute to higher incidences of mental health disorders and other health concerns. Minority stress specifically addresses the excessive stress experienced by individuals from stigmatized social categories as a result of their marginalized social identities (i.e., race, religion, sexual orientation, gender identity), which deviate from societal norms and influence intergroup relations. ${ }^{6}$

Viewing minority stress through the lens of the model of multiple dimensions of identity highlights how different identity dimensions are present in each individual but vary in degree of saliency based on context. Common moderators of salience are family background, sociocultural conditions, and current life experiences. 9 The minority stress processes include the experience of prejudice, expectations of being rejected, hiding and concealing, internalized homophobia, and various coping processes. ${ }^{6}$ Since identity is both externally defined and internally experienced,

Copyright $(\subset)$ by Indiana State University All rights reserved. ISSN Online 2577-8188 instances of discrimination or expectations imposed from outside sources heavily impacts how a person makes meaning of their life experiences. 9 The cumulative effect of holding a minority position in society further exacerbates the unyielding cycle of stress. In an attempt to cope, African-American LGBTQ+ persons may compartmentalize their sexuality or attempt to diminish or hide their sexuality so they are seen embodying only one marginalized identity. Hiding this aspect of their identity may help to lessen the anticipated effects of minority stress but will likely worsen their health outcomes.' Although taxing, the process of hiding an invisible, marginalized identity and engaging in performativity of gender occurs in the hope of receiving more equitable care. As noted earlier, a negative experience with a healthcare provider may result in a lack of trust in healthcare providers and lead an individual to not seek care.

One way to alleviate the stress placed upon vulnerable patients is to create a safe space and engage in shared decision-making, which may facilitate better patient-provider communication and treatment compliance. A safe space can be anywhere that individuals feel included and comfortable being themselves. For example, in an inclusive athletic training clinic, a patient that identifies as transgender can ask the athletic trainer and others to refer to them by the pronouns with which they identify without negative consequence. An inclusive healthcare facility uses inclusive intake forms that allow patients to share their identity and guide patient care. A safe space allows patients to share relevant medical information freely, without judgement, as part of the shared decision-making process ${ }^{1}$ In addition to creating a safe space, clinicians must maintain awareness of the disadvantages among patients of marginalized identities within the healthcare system and across social class while providing patient-centered care for all. ${ }^{1}$ A lack of a safe space by a healthcare provider can lead to disadvantages experienced by minoritized 
groups; these may include barriers to access, erasure of identity (gender identity, sexual orientation), healthcare stereotype threat, and refusal to treat, which lead to healthcare disparities.

\section{HEALTHCARE DISPARITIES}

In addition to the minority stress experienced by individuals in marginalized groups, these individuals often experience health disparities, or a diminished quality of health and healthcare across racial, ethnic, and socioeconomic groups. 1,5 Healthcare disparities often occur as a result of explicit or implicit biases from their healthcare provider, often affecting both mental and physical health. For example, an athletic trainer may have negative biases toward individuals that are transgender; as a result, the clinician may not provide adequate care to a transgender patient that is injured. These implicit biases by clinicians toward sexual and gender minorities, as well as other marginalized groups, have been shown to negatively impact patient perception of care, clinical decisions, and patient-clinician interactions. ${ }^{3,10}$ These disparities have been noted in various settings, including the pediatric population. LGBTQ+ youth are at greater risk of healthcare disparities compared to their heterosexual, cisgender counterparts. ${ }^{11}$ These inequities may be due to stigmatization, family rejection, victimization, and lack of adequate training in healthcare providers.11 The disadvantages faced in LGBTQ+ youth often continue into adulthood and can lead to increased substance abuse, depression, anxiety, and suicide. ${ }^{11}$

Research on healthcare disparities often focuses on single social groups, and not on the intersection of various identifiers or groups an individual may belong to. ${ }^{1}$ The intersection of gender and sexual minorities with race and ethnicity may result in greater healthcare disparities for individuals that may be subjected to discrimination due to

Copyright (C) by Indiana State University All rights reserved. ISSN Online 2577-8188 belonging to multiple marginalized groups. This is especially true for BIPOC that identify as being part of the LGBTQ+ community.

Although recent studies suggest athletic trainers are knowledgeable about the importance of patient-centered care and being culturally competent, it is unclear how clinicians can best implement this knowledge into clinical practice. Athletic trainers and other healthcare providers should strive to continue to learn more about cultural competency and how to incorporate this information into their history taking, physical exams, management of injuries, and other aspects of their clinical practice. Some examples of how athletic trainers can learn more and be more inclusive include seeking out continuing education opportunities regarding the LGBTQ+ population and healthcare as well as reading the latest research regarding this patient population.

Athletic trainers and other clinicians can utilize this information to create a more inclusive environment in their setting. Creating an inclusive athletic training environment can include the use of appropriate terminology and representation of inclusion and diversity, creating culturally competent forms for documentation, and ensuring all policies and procedures are reviewed to ensure they are inclusive (Figure 1).12 It is also important to assess environmental and social factors that may be impacting the patient's physical and mental health. ${ }^{1}$ Whether or not an LGBTQ+ individual is accepted by their family, friends and teammates, for example, can impact an individual's physical and mental health status. If a young high school athlete has been kicked out of his home and does not have access to basic needs or medical insurance, this can have a devastating impact on their health and wellbeing. Incorporating this information into the care provided would be a prime example of how minority stress and the negative impacts that discrimination and negative experiences can have 
on someone's physical and mental health can impact the care rendered by the athletic trainer.

\section{CLINICAL APPLICATION}

There are several action steps providers can take to ensure that they are providing patient-centered care to all patients, particularly those that are BIPOC, of sexual or gender minorities or other marginalized groups. Patient-centered care is a philosophy of care that encourages shared decision-making, focusing on the patient as a whole, and treats patients as partners in care. By understanding the role intersectionality plays in the lives of some of our patients, athletic trainers will enhance the patient-provider relationship. Assessing one's own biases will provide insight of how care may need to be adjusted to meet a patient's needs. Research has demonstrated disparities in shared decision-making and communication among sexual and gender minorities as well as BIPOC and other racial/ethnic disparities. Therefore, it is imperative for healthcare providers to ensure they are utilizing shared decision-making and patientcentered care. Through shared decision-making, the clinician and patient can work together to have open communication, discuss treatment options, and develop a plan that will meet the needs of the patient. ${ }^{1}$ In addition to a selfassessment and the individual initiatives of each clinician, there are several system-based actions that should be taken to improve the clinic environment. The clinic should publicly display their non-discrimination policy, provide diverse educational materials, use inclusive intake forms, and train the entire staff (i.e., receptionist, insurance coordinators, etc.) not just the clinicians. Furthermore, the facility should perform regular audits to ensure that the patients' experience match the intended outcomes of the clinic.

\section{CONCLUSION}

Most LGBTQ+ and BIPOC individuals have experienced some form of discrimination in their lives. Individuals that identify both as LGBTQ+ and BIPOC likely experience more discrimination due to the intersection of their race and sexual orientation or gender identity. Unfortunately, some of these instances of discrimination have occurred with healthcare providers. As athletic trainers, it is important for us to increase our knowledge of the impact of intersectionality and minority stress on healthcare, examine our own biases, create an inclusive athletic training setting, and ensure that we are providing quality patientcentered care to all patients.

\section{REFERENCES}

1. Peek ME, Lopez FY, Williams HS, et al. Development of a conceptual framework for understanding shared decision making among African-American LGBT patients and their clinicians. J Gen Intern Med. 2016;31:677687. https://doi.org/10.1007/s1 1606-0163616-3.

2. Crenshaw K. Demarginalizing the intersection of race and sex: A black feminist critique of antidiscrimination doctrine, feminist theory and antiracist politics. $U$ Chi Legal $F$. 1989:139-168.

3. Arnold $E$, Dhingra N. Health care inequities of sexual and gender minority patients. Dermatol Clin. 2020;38:185-190. https://doi.org/10.1016/i.det.2019.10.002

4. Ensign KA, Yiamouyiannis $A$, White KM, Ridpath BD. Athletic trainers' attitudes toward lesbian, gay, and bisexual National Collegiate Athletic Association studentathletes. J Athl Train. 2011;46: 69-75. https://doi.org/10.4085/1062-6050-26017.

5. Nye EA, Crossway A, Rogers SM, Games KE, Eberman LE. Lesbian, gay, bisexual, transgender, and queer patients: Collegiate athletic trainers' perceptions. J Athl Train. 2019;54:334-344.

Clinical Practice in Athletic Training Volume 4 - Issue 1 - February 2021 
https://doi.org/10.4085/1062-6050-260-

17.

6. Meyer IH. Prejudice, social stress, and mental health in lesbian, gay, and bisexual populations: Conceptual issues and research evidence. Psychol Bull. 2003;129:674-697. https://dx.doi.org/10.1037\%2F00332909.129.5.674.

7. Bowleg L. The problem with the phrase women and minorities: Intersectionality-an important theoretical framework for public health. Am J Public Health. 2012;102:1267-1273. https://doi.org/10.2105/AJPH.2012.30075 $\underline{0}$.

8. Hankivsky O. Women's health, men's health, and gender and health: Implications of intersectionality. Soc Sci Med. 201 2;74:17121720.

https://doi.org/10.1016/i.socscimed.2011.1 1.029.

9. Jones SR, McEwen MK. A conceptual model of multiple dimensions of identity. J Coll Stud Dev. 2000;41:405-414.

10. McDowell MJ, Goldhammer H, Potter JE, Keuroghlian AS. Strategies to mitigate clinician implicit bias against sexual and gender minority patients. Psychosomatics. 2020; 61(6): 655-661. https://doi.org/10.1016/i.psym.2020.04.0 21.

11. Hafeez $H$, Zeshan $M$, Tahir MA, Jahan $N$, Naveed S. Health care disparities among lesbian, gay, bisexual, and transgender youth: A literature review. Cureus. 2017;9:e 1184.

https://doi.org/10.7759/cureus.1 184.

12. Harriell K. Teaching, learning cultural competency: Respecting patient values, preferences vital to patient-centered care. In: NATA News. vol. April 2020: 18-19. 


\section{Intersectionality and the Role of the Athletic Trainer to Provide Culturally Competent Patient- Centered Care}

\section{Intersectionality}

A theoretical framework that considers multiple social categories (e.g., race, ethnicity, gender, sexual orientation, socioeconomic status) intersecting at the micro level of individual experience to reflect multiple interlocking systems of privilege and oppression at the macro, social-structural level (e.g., racism, sexism, heterosexism).

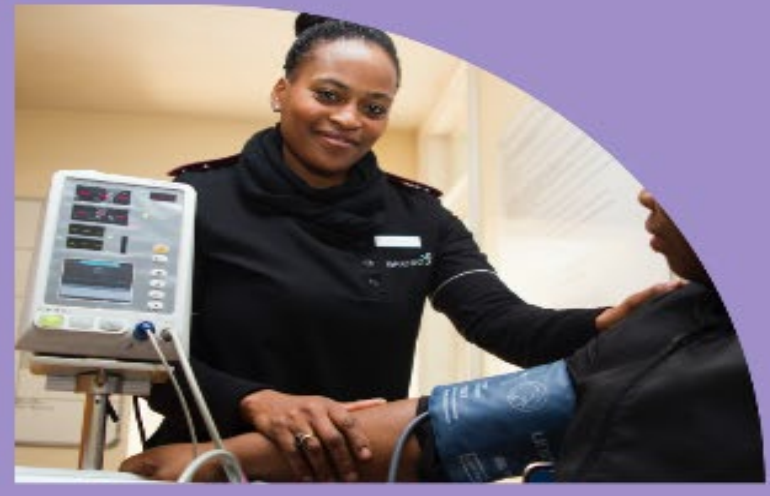

\section{Shared Decision- Making}

When patients and clinicians work collaboratively to identify treatment plans that meet the individual patient needs and preferences.

The shared decision-making model has 3 key domains:

- Information-sharing between patients and providers

- Deliberation about the pros and cons of treatment choices

- Decision making about a treatment plan that is endorsed by both the patient and the clinician.

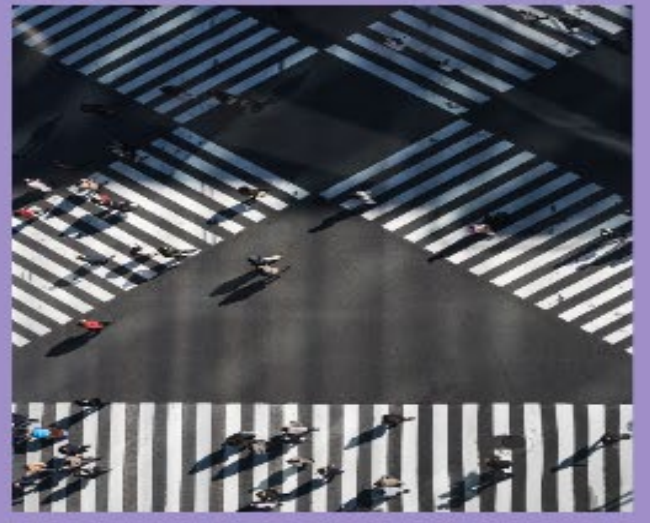

\section{Patient-Centered Care}

Care that is respectful of, and responsive to, individual patient preferences, needs and values, and ensuring that patient values guide all clinical decisions. By providing patient-centered care a clinician considers the interactive effect of multiple identities and how the cumulative lived experience of individuals contributes to health disparities.

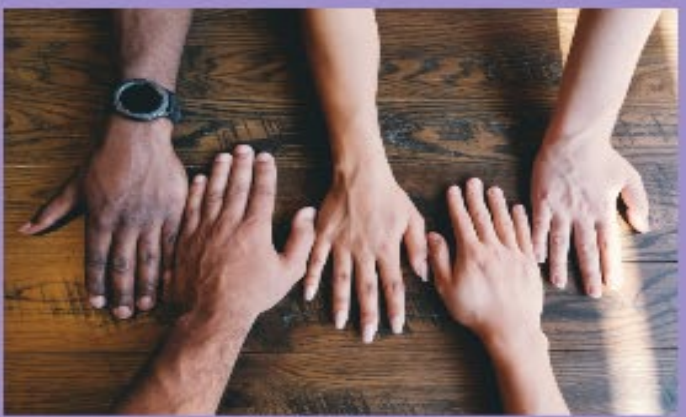

\section{Figure 1: Supplemental Infographic}

Copyright (C) by Indiana State University All rights reserved. ISSN Online 2577-8188 Volume 4 - Issue 1 - February 2021 


\section{Impact on Healthcare and Outcomes}

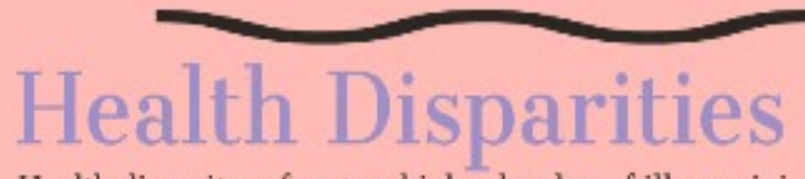

Health disparity refers to a higher burden of illness, injury, disability, or mortality experienced by one group relative to another.

Currently health disparities are not well studied through the lens of intersectionality but rather single societal groups. Minority stress is the unique stress that sexual minority people experience as a result of 'stigma, prejudice, and discrimination', but overall health disparities are not well

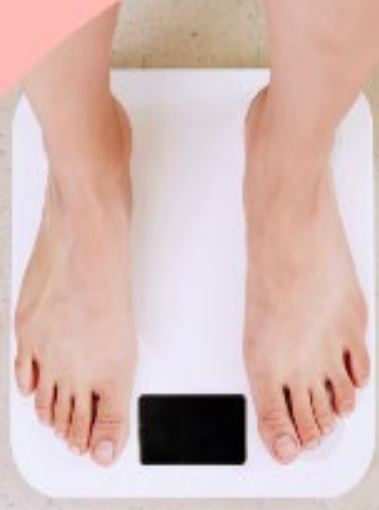
studied through the lens on intersectionality

For example, a gay African-American male will have interactive effects of being gay and African-American as those identities intersect. This will be different for a male that is either gay or African-American.
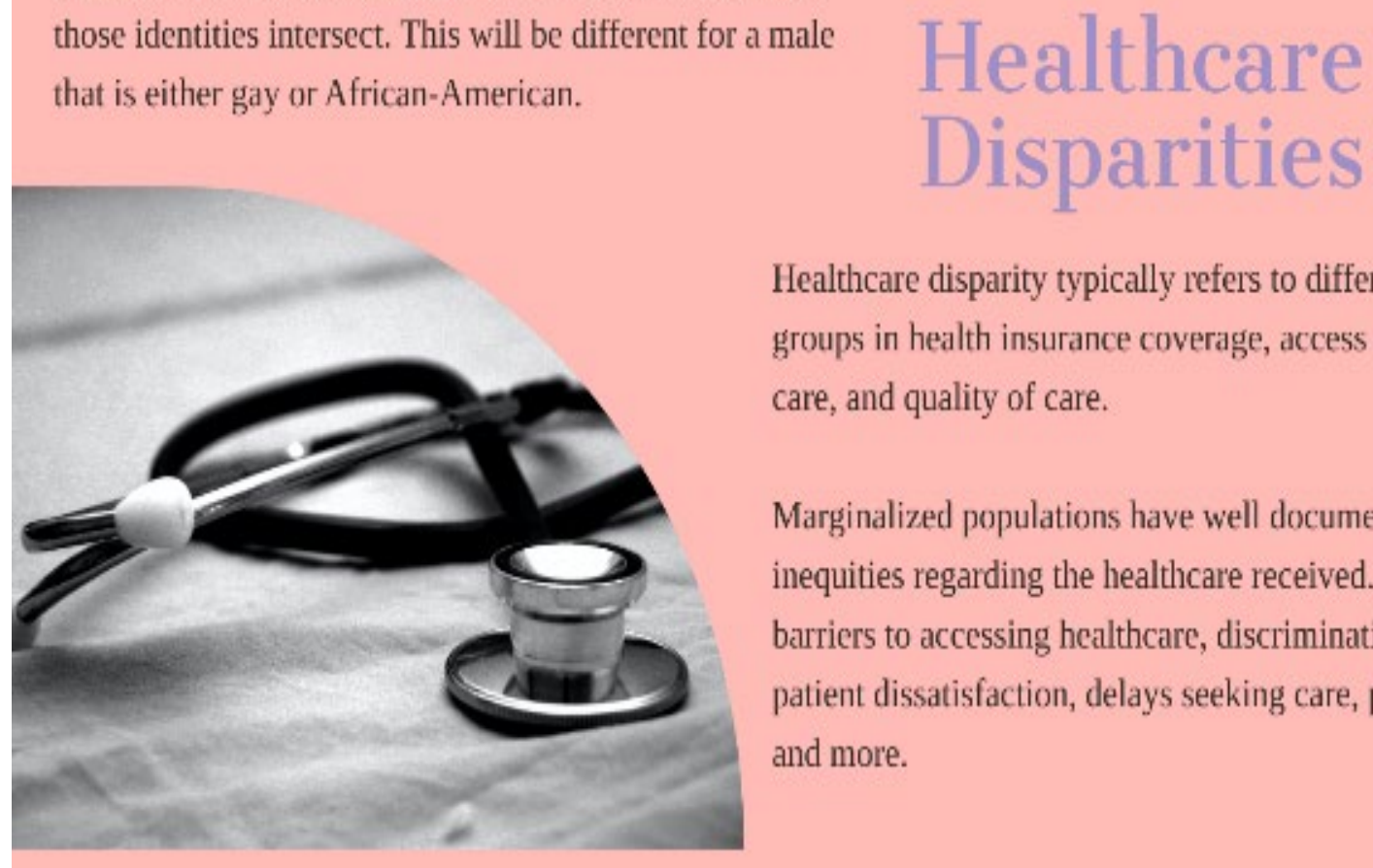

Healthcare disparity typically refers to differences between groups in health insurance coverage, access to and use of care, and quality of care.

Marginalized populations have well documented and studied inequities regarding the healthcare received. This includes barriers to accessing healthcare, discrimination, biases, patient dissatisfaction, delays seeking care, poorer outcomes and more. 


\section{Action Items For Athletic Trainers}

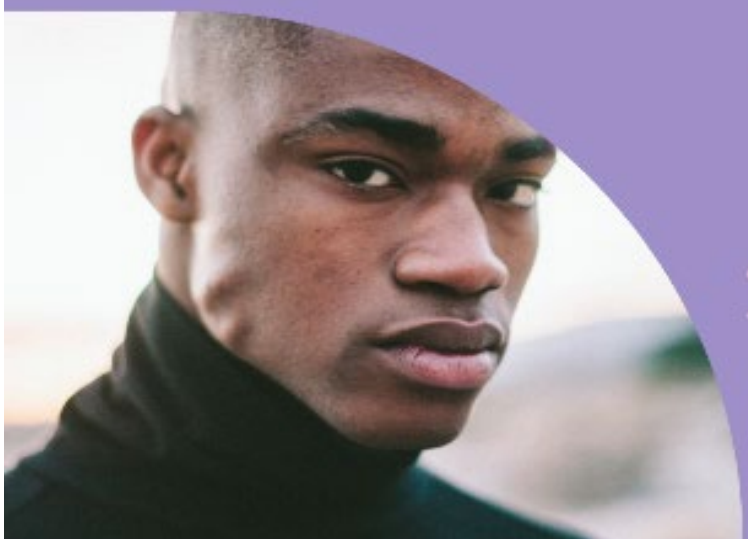

\section{Know your patients}

Spend time getting to know your patients. Identity is both externally and internally defined. Don't make assumptions and engage your patient in their healthcare.

\section{Assess your biases}

External (explicit) bias are the stereotypes or prejudices toward one group that you are conscious of, whereas internal (implicit) biases are the biases you are unaware you have as a clinician. Both have a significant impact on health care.

\section{Use Inclusive Intake Forms}

Allow the patient the opportunity to share their iclentities with the clinician and use the information to guide patient care.

\section{Implement Shared Decision-Making}

Work collaboratively with your patient to decide on plan of care. This will improve patient compliance, adherence, and comprehension.

\section{Audit Your Practice}

Audit your documentation to see if you are accounting for your interactions, and use patient satisfaction survey. Are your patients

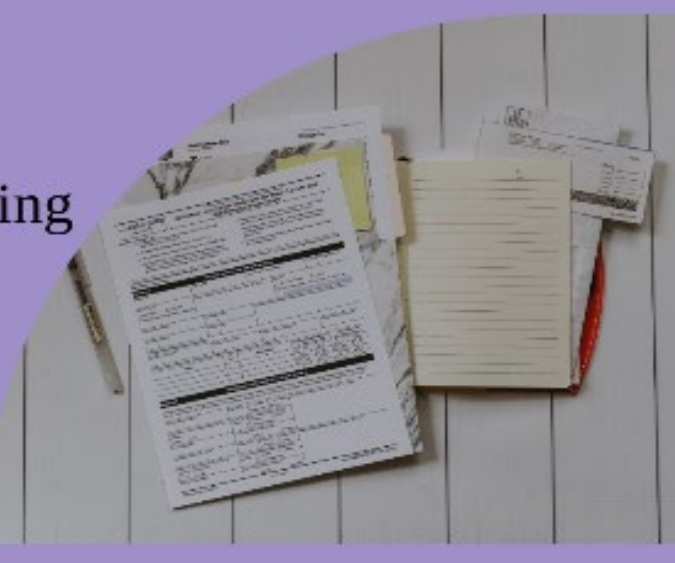
experiences the same as you perceive them?

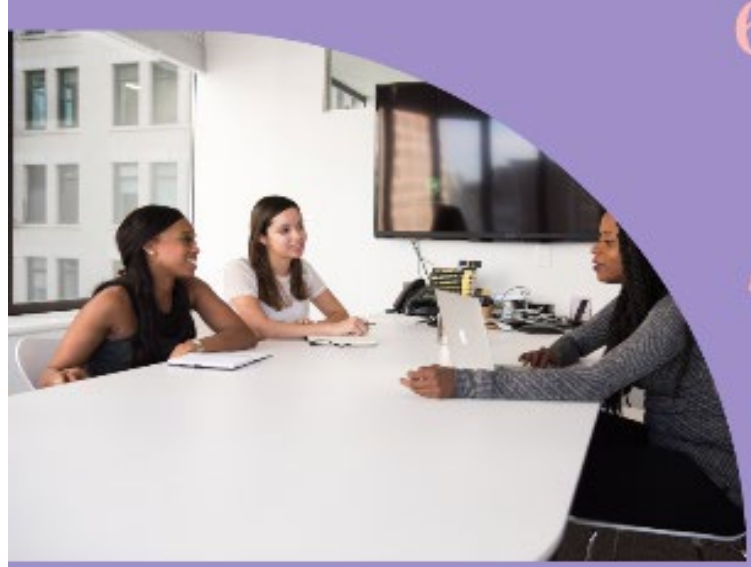

\section{Create Inclusive Facility}

Publicly display an inclusive non-discrimination policy. Provide educational materials that are diverse including sexual orientation, race, ethnicity, age, etc.

\section{Staff Training}

All staff should be trained to create a welcoming and inclusive experience in the clinic. Staff should be mindful of using inclusive language, non-presumptive interviewing, and educated on important health related conditions for all marginalized populations.

Clinical Practice in Athletic Training 